\title{
Editorial
}

\section{Biosocial Strategies to Address the Socioeconomic Determinants and Consequences of the TB and COVID-19 Pandemics}

\author{
Debora Pedrazzoli ${ }^{1 \star}$ and Tom Wingfield ${ }^{2,3,4}$ \\ ${ }^{1}$ Department of Infectious Disease Epidemiology, London School of Hygiene and Tropical Medicine, London, United Kingdom; ${ }^{2}$ LIV-TB Lead, \\ Departments of Clinical Sciences and International Public Health, Liverpool School of Tropical Medicine, Liverpool, United Kingdom; ${ }^{3}$ Tropical and \\ Infectious Disease Unit, Liverpool University Hospitals NHS Foundation Trust, Liverpool, United Kingdom; ${ }^{4}$ Department of Global Public Health, \\ Karolinska Institutet, Stockholm, Sweden
}

COVID-19 and tuberculosis (TB) are damaging, dual pandemics, which are more than mere health crises; they are socioeconomic and humanitarian crises that require a biosocial response..$^{1,2}$

The socioeconomic determinants of TB and COVID-19 are pernicious and overlapping. Poverty, overcrowded housing conditions, under- or malnutrition, chronic comorbidities such as lung disease and diabetes, and belonging to marginalized, underserved communities and minority ethnic groups are all key determinants. ${ }^{3}$ COVID-19 has distorted health systems at all levels. It has contracted clinical services, decimated staffing levels, reconfigured laboratories including the repurposing of GeneXpert modules for TB diagnosis, rolled back hard won progress toward Universal Health Coverage (UHC) and global health security, ${ }^{4}$ and diverted much-needed resources away from other diseases, including TB. ${ }^{5,6}$ As a result, the COVID19 pandemic is predicted to lead to a $20 \%$ increase in TB deaths in high-burden countries over the next 5 years. $^{7}$

The health and socioeconomic consequences of TB and COVID-19 are highly harmful and inequitably distributed. ${ }^{8}$ Impoverished individuals, households, and communities continue to be disproportionately affected by both TB and COVID-19. Social distancing and isolation measures, restricted movement and quarantine, illness, and care-seeking impose a severe socioeconomic burden, especially on those who are unemployed, in the informal job sector, or lack adequate social protection. ${ }^{1}$ These factors are not only associated with impaired healthcare access and worse health outcomes $^{9,10}$; they can push those affected into further impoverishment, typifying the "medical poverty trap."11 The corrosive influence of COVID-19 and its related mitigation strategies on mental health and domestic violence is a parallel, concomitant emergency. ${ }^{12}$

Far from being great "levellers," these intersecting pandemics have reemphasized intolerable and persistent global inequalities in health, wealth, and well-being-inequalities that are aggravated by poverty of voice, agency, and opportunity. ${ }^{13}$ However, the convergent challenges brought about by COVID-19 and TB enable us to identify potential opportunities to mitigate their impact.

Key to this is collaboration toward achieving the interlaced WHO Sustainable Development Goals (SDGs)—specifically SDG1 (No poverty), SDG2 (Zero hunger), SDG3 (Good health and well-being), SDG8 (Decent work and economic growth),

${ }^{*}$ Address correspondence to Debora Pedrazzoli, Department of Infectious Disease Epidemiology, London School of Hygiene and Tropical Medicine, Keppel Street, London WC1E 7HT, United Kingdom. E-mails: debora.pedrazzoli@Ishtm.ac.uk or pedrazzolid@who.int and SDG10 (Reducing inequalities). ${ }^{14}$ Modeling studies have suggested that eradicating poverty and providing social protection would reduce TB incidence by more than $84 \%$ by $2035^{15}$ - similar modeling studies would be of benefit when applied to COVID-19. Moreover, in the WHO End TB Strategyfor the first time in the modern era of TB control-we were provided with an ambitious socioeconomic goal that "zero TB-affected families face catastrophic costs" by $2025 .^{16}$ In reality, catastrophic costs mitigation should be a global goal regardless for all states of ill health, whether mental or physical, noncommunicable or communicable, occupational or accidental, COVID-19 or TB.

The article by Fuady et al. ${ }^{17}$ in this issue of the American Journal of Tropical Medicine and Hygiene examining the impact of COVID-19 on costs incurred by people with TB and their households is topical, timely, and moves this field forward. The article draws attention to this overlooked area of research, policy, and practice, and provides an informative conceptual framework that will help us to develop better biosocial responses to combat both TB and COVID-19.

So, how can we address the socioeconomic determinants and consequences of TB and COVID-19, and prevent what our colleagues have rightly called the "perfect storm?"18

First, there is an urgent need to better understand the socioeconomic impacts of COVID-19, especially in the most vulnerable groups. Evidence from nationally representative surveys of costs incurred by people with TB and their households (known as "TB Patient Cost Surveys") shows that more than one in two TB-affected households worldwide incur catastrophic costs. ${ }^{19}$ These surveys and related mixed-methods research also show that significant drivers of catastrophic costs are: lost income and time, reduced productivity, and significant non-medical expenses such as travel to access healthcare services and nutritional expenditure to meet even basic food requirements. ${ }^{20-22}$ In certain regions, these costs may be exacerbated by an unregulated and convoluted public-private sector $\mathrm{mix}^{23}$ and avoidable expenditure on unproven, costly "therapies" including nutritional supplements. Furthermore, mask-wearing and respiratory symptoms of COVID-19 and TB may be associated with diagnostic uncertainty, stigma, discrimination, and isolation, which further compound challenges to accessing care and appropriate treatment. ${ }^{18,24}$ We have the perfect opportunity now to measure and evaluate the socioeconomic impacts along the care-seeking pathway for people with acute, chronic, or acute-on-chronic respiratory symptoms.

Second, understanding the patient pathway will provide valuable information to inform assessment of the progress made toward the SDGs. This is particularly pertinent with 
relation to achieving SDG3 and UHC but also to the underacknowledged yet acute need to expand social protection coverage and establish basic universal social protection floors. ${ }^{25,26}$ National lockdown, "stay at home," and "shelter in place" policies have already had a detrimental effect on individual and household incomes and been associated with contractions in national gross domestic product indices. ${ }^{14}$ There is a clear negative association between incidence and mortality of TB and proportional governmental expenditure on social protection. ${ }^{27,28}$ It is as yet unclear whether the same association exists with relation to COVID-19, but that evidence will be vital to shape our response.

Third, despite their detrimental effects, large-scale nonpharmacological interventions may also provide a useful opportunity to explore ways in which we can mitigate careseeking costs of TB, COVID-19, and other poverty-related diseases. For example, measures that reduce the need for daily encounters between TB patients and healthcare staff to reduce COVID-19 transmission have been rapidly rolled out and could be further strengthened. ${ }^{29}$ One such measure is the WHO recommendation of all-oral TB treatment regimens for multidrug- and rifampicin-resistant TB and extensively drugresistant $\mathrm{TB}^{30}$ This strategy can lower costs for people with TB and their households through reduction of direct costs associated with traveling to health facilities for daily injections, and/or opportunity costs associated with lost productivity and income due to hospitalization during the initial "intensive" phase of treatment. ${ }^{31}$ Similarly, intensified use of digital health technologies, such as video observed therapy and electronic medication monitors to support programs and affected people could pave the way to models of care that may lower medical, non-medical, and opportunity costs even further. ${ }^{32}$

Fourth, the COVID-19 pandemic has brought into sharp relief the weaknesses of health systems that are imbalancedin terms of both funding and focus-toward secondary care. We could use this opportunity to garner feedback from users of the health system on how best to improve the care we provide not only in secondary care but at all levels. ${ }^{33}$ This is also an opportune moment to reconsider how best to engage communities and community health workers to harness their commitment, knowledge, trust, and understanding of their local areas. ${ }^{34}$ Outreach involving peer support and advocacy is a much needed vehicle of change to not only reduce careseeking costs by bringing care to people's door in the community but also to use peer-led education and information as a tool to promote agency, to empower, and to combat stigma. ${ }^{35}$ In this way, we could ensure no one is left behind and eradicate the misplaced blame attributed to the so-called "hard-to-reach" groups who, in reality, are underserved by static and fragmented local health and social care systems. ${ }^{36}$

Finally, building on the aforementioned, this is the time to move away from vertical public health programs. We need health systems that are responsive to the needs of vulnerable communities and resilient to the threat of infections, especially those which are airborne. A major step toward reaching this goal would be inclusive horizontal approaches that integrate biomedical strategies to prevent and tackle the health impacts of syndemics with biosocial strategies to address their determinants and consequences. ${ }^{37}$ People affected by povertyrelated illnesses, including respiratory illnesses, would benefit vastly from this joined-up approach.
The COVID-19 pandemic is threatening decades of progress made in TB control and, undoubtedly, continues to have the biggest impact on people and communities affected by TB and poverty. Predicted increases in levels of food insecurity, rising impoverishment, and contraction of gross domestic product have led to cross-sectoral bodies, including the WHO, academia, and civil society, to ring the alarm bell. ${ }^{38}$ Findings from ongoing and future TB Patient Cost Surveys will provide an accurate estimate of the socioeconomic impact of COVID19 on people with TB and their households. The surveys will simultaneously provide critical data to inform broader mapping of social determinants and consequences of ill health, uptake and coverage of social protection strategies and $\mathrm{UHC}$, and therefore progress toward the SDGs.

As we begin what we hope will be a brighter 2021, we must turn the challenges of the COVID-19 and TB pandemics into an opportunity to refine biosocial strategies that not only improve health and well-being but also address poverty and inequality.

Received December 23, 2020. Accepted for publication December 25, 2020.

Published online January 6, 2021.

Financial support: T. W. is supported by grants from the Wellcome Trust, UK (209075/Z/17/Z); the Medical Research Council, Department for International Development, and Wellcome Trust (Joint Global Health Trials, MR/V004832/1), the Academy of Medical Sciences, UK; and the Swedish Health Research Council, Sweden.

Authors' addresses: Debora Pedrazzoli, Department of Infectious Disease Epidemiology, London School of Hygiene and Tropical Medicine, London, United Kingdom, E-mails: debora.pedrazzoli@ Ishtm.ac.uk or pedrazzolid@who.int. Tom Wingfield, LIV-TB Lead, Departments of Clinical Sciences and International Public Health, Liverpool School of Tropical Medicine, Liverpool, United Kingdom, Tropical and Infectious Disease Unit, Liverpool University Hospitals NHS Foundation Trust, Liverpool, United Kingdom, and Department of Global Public Health, Karolinska Institutet, Stockholm, Sweden, E-mail: tom.wingfield@Istmed.ac.uk.

This is an open-access article distributed under the terms of the Creative Commons Attribution (CC-BY) License, which permits unrestricted use, distribution, and reproduction in any medium, provided the original author and source are credited.

\section{REFERENCES}

1. The Lancet, 2020. COVID-19 in Latin America: a humanitarian crisis. Lancet 396: 1463. doi:10.1016/S0140-6736(20)32328-X.

2. Ortblad KF, Salomon JA, Bärnighausen T, Atun R, 2015. Stopping tuberculosis: a biosocial model for sustainable development. Lancet 386: 2354-2362.

3. Wingfield T et al., 2016. Beyond pills and tests: addressing the social determinants of tuberculosis. Clin Med 16: S79-S91.

4. Lal A, Erondu NA, Heymann DL, Gitahi G, Yates R, 2021. Fragmented health systems in COVID-19: rectifying the misalignment between global health security and universal health coverage. Lancet 397: 61-67. doi: 10.1016/S0140-6736(20) 32228-5.

5. Venkatesan $P, 2020$. COVID-19 diagnostics-not at the expense of other diseases. Lancet Microbe 1: e64.

6. World Health Organization, 2020. The Impact of the COVID-19 Pandemic on Noncommunicable Disease Resources and Services: Results of a Rapid Assessment. Geneva, Switzerland: WHO. Report No.: 9240010297.

7. Hogan AB et al., 2020. Potential impact of the COVID-19 pandemic on HIV, tuberculosis, and malaria in low-income and middle-income countries: a modelling study. Lancet Glob Health 8: e1132-e1141. 
8. Wingfield T, Cuevas LE, MacPherson P, Millington KA, Squire SB, 2020. Tackling two pandemics: a plea on World Tuberculosis Day. Lancet Respir Med 8: 536-538.

9. Patel J, Nielsen F, Badiani A, Assi S, Unadkat V, Patel B, Ravindrane R, Wardle H, 2020. Poverty, inequality and COVID19: the forgotten vulnerable. Public Health 183: 110.

10. Migliori GB et al., 2020. Worldwide effects of coronavirus disease pandemic on tuberculosis services, January-April 2020. Emer Infect Dis 26: 2709.

11. Wingfield T, Boccia D, Tovar M, Gavino A, Zevallos K, Montoya R, Lönnroth K, Evans CA, 2014. Defining catastrophic costs and comparing their importance for adverse tuberculosis outcome with multi-drug resistance: a prospective cohort study, Peru. PLoS Med 11: e1001675.

12. Hamadani JD et al., 2020. Immediate impact of stay-at-home orders to control COVID-19 transmission on socioeconomic conditions, food insecurity, mental health, and intimate partner violence in Bangladeshi women and their families: an interrupted time series. Lancet Glob Health 8: e1380-e1389.

13. Tran PB et al., 2020. Income security during public health emergencies: the COVID-19 poverty trap in Vietnam. BMJ Glob Health 5: e002504.

14. Lönnroth K, Raviglione M, 2016. The WHO's new End TB strategy in the post-2015 era of the sustainable development goals. Trans R Soc Trop Med Hyg 110: 148-150.

15. Carter DJ et al., 2018. The impact of social protection and poverty elimination on global tuberculosis incidence: a statistical modelling analysis of Sustainable Development Goal 1. Lancet Glob Health 6: e514-e522.

16. World Health Organization, 2015. The End TB Strategy. Geneva, Switzerland: WHO.

17. Fuady A, Houweling TAJ, Richardus JH, 2020. COVID-19 and tuberculosis-related catastrophic costs. Am J Trop Med Hyg (Epub ahead of print 2 Dec 2020). doi: 10.4269/ajtmh.20-1125.

18. Saunders MJ, Evans CA, 2020. COVID-19, tuberculosis, and poverty: preventing a perfect storm. Eur Respir J 56: 2001348. doi: 10.1183/13993003.01348-2020.

19. World Health Organization, 2020. Global Tuberculosis Report 2020. Geneva, Switzerland: WHO.

20. Wingfield T, Tovar MA, Huff D, Boccia D, Montoya R, Ramos E, Lewis JJ, Gilman RH, Evans CA, 2016. The economic effects of supporting tuberculosis-affected households in Peru. Eur Respir J 48: 1396-1410.

21. Tanimura T, Jaramillo E, Weil D, Raviglione M, Lönnroth K, 2014. Financial burden for tuberculosis patients in low- and middle-income countries: a systematic review. Eur Respir J 43: 1763-1775.

22. Gurung SC et al., 2019. The role of active case finding in reducing patient incurred catastrophic costs for tuberculosis in Nepal. Infect Dis Pov 8: 99.

23. Rai B et al., 2020. Developing feasible, locally appropriate socioeconomic support for TB-affected households in Nepal. Trop Med Infect Dis 5: 98.
24. Villa S, Jaramillo E, Mangioni D, Bandera A, Gori A, Raviglione MC, 2020. Stigma at the time of the COVID-19 pandemic. Clin Microbiol Infect. 26: 1450-1452.

25. Lönnroth K, Tessier L, Hensing G, Behrendt C, 2020. Income security in times of ill health: the next frontier for the SDGs. BMJ Global Health 5: e002493.

26. Thorpe J, Viney K, Hensing G, Lönnroth K, 2020. Income security during periods of ill health: a scoping review of policies, practice and coverage in low-income and middle-income countries. BMJ Global Health 5: e002425.

27. Siroka A, Ponce NA, Lönnroth K, 2016. Association between spending on social protection and tuberculosis burden: a global analysis. Lancet Infect Dis 16: 473-479.

28. Reeves A, Basu S, McKee M, Stuckler D, Sandgren A, Semenza J, 2014. Social protection and tuberculosis control in 21 European countries, 1995-2012: a cross-national statistical modelling analysis. Lancet Infect Dis 14: 1105-1112.

29. World Health Organization, 2020. COVID-19: Considerations for Tuberculosis (TB) Care. Geneva, Switzerland: WHO.

30. World Health Organization, 2020. Consolidated Guidelines on Tuberculosis: Module 4: Treatment: Drug-Resistant Tuberculosis Treatment. Geneva, Switzerland: WHO.

31. Special Programme for Research and Training in Tropical Diseases, 2020. ShORRT Research Package. Available at: https://www. who.int/tdr/research/tb_hiv/shorrt/en/2019. Accessed December 22, 2020.

32. World Health Organization, 2015. Digital Health for the End TB Strategy: an Agenda for Action. Geneva, Switzerland: WHO.

33. Wu M-S, Hayat F, Ratcliffe L, Beadsworth MB, Defres S, Wingfield T, 2020. What do people hospitalised with COVID-19 think about the care they received? BMJ 370: $\mathrm{m} 3496$.

34. Saunders M, Koh G, Small A, Dedicoat M, 2014. Predictors of contact tracing completion and outcomes in tuberculosis: a 21-year retrospective cohort study. Int J Tuberc Lung Dis 18: 640-646.

35. Wingfield T, Tovar MA, Huff D, Boccia D, Montoya R, Ramos E, Datta S, Saunders MJ, Lewis JJ, Gilman RH, Evans CA, 2017. A randomized controlled study of socioeconomic support to enhance tuberculosis prevention and treatment, Peru. Bull World Health Organ 95: 270.

36. Heuvelings CC et al., 2017. Effectiveness of interventions for diagnosis and treatment of tuberculosis in hard-to-reach populations in countries of low and medium tuberculosis incidence: a systematic review. Lancet Infect Dis 17: e144-e158.

37. Singer M, Bulled N, Ostrach B, Mendenhall E, 2017. Syndemics and the biosocial conception of health. Lancet 389: 941-950.

38. SPARKS Network, 2020. Bridging the SDGs to Optimise HealthRelated Social Protection. Available at: https://sparksnetwork.ki.se/ about-sparks-conference/2020. Accessed December 22, 2020. 